\section{REVISTA BRASILEIRA DE QUALIDADE DE VIDA}

\title{
Avaliação da qualidade de vida após programa de intervenção direcionado à melhora da qualidade do sono
}

\section{Quality of life assessment after intervention program related to the improvement of the quality of sleep}

\author{
Isaac Rosa Marques \\ Universidade de Santo Amaro - UNISA - São Paulo - SP - Brasil \\ imarques@unisa.br \\ Ricardo de Oliveira Isabel \\ Universidade de Santo Amaro - UNISA - São Paulo - SP - Brasil \\ rihannibal@hotmail.com
}

\begin{abstract}
Resumo
O estudo teve como objetivo avaliar a qualidade de vida (QV) de pacientes portadores de Síndrome da Apneia e Hipopneia Obstrutiva da Sono (SAHOS) após um programa de intervenção. Tratou-se de um estudo quase-experimental do tipo antes-depois realizado com dois grupos - Grupo Controle (GC) e Grupo Experimental (GE) - de nove pacientes portadores de SAHOS, sendo o GE submetido a um programa de intervenção e ambos avaliados com o instrumento SF-36. Os resultados evidenciaram melhora estatisticamente significante na QV do GE. O teste de análise de variância de Friedman apresentou os seguintes resultados: GC 5,09 ( $\mathrm{p}=0,6490)$ e GE 11,7 ( $\mathrm{p}=0,1109)$. Concluiu-se que após a intervenção sobre higine do sono houve melhora na QV relacionada à melhora da qualidade do sono.
\end{abstract}

Palavras-chave: distúrbios do sono, estudos de intervenção, qualidade de vida.

\section{Abstract}

The purpose of the study was to assess the quality of life (QoL) of patients with the Apnea Syndrome and Obstructive Sleep Hypopnea (ASOSH) after an intervention program. That was a quasi-experimental study type before-after carried out with two groups - Control Group (CG) and Experimental Group (EG) - constituted by nine patients with ASOSH each group, being the EG submitted to an intervention program and both assessed with the SF-36. Results demonstrated statistically significant improvement in QoL of the EG. Freidman's variance analysis presented the following results: CG 5,09 ( $\mathrm{p}=0,6490)$ and EG $11.7(\mathrm{p}=0,1109)$. It was concluded that after the intervention about sleep hygiene improvement in QoL related to the improvement of quality of sleep was verified.

Keywords: sleep disorders, intervention studies, quality of life.

\section{Introdução}

A Síndrome da Apneia e Hipopneia Obstrutiva do Sono (SAHOS) é definida como episódios repetitivos de oclusão das vias aéreas superiores durante o período do sono, associado a 
fragmentação do sono. Quando associada à sonolência excessiva e/ou a doença cardiovascular é considerada síndrome (PAKOLA, 1995).

A SAHOS é muito comum na população em geral. Em estudo populacional de adulto em idade média (30 a 60 anos), foi encontrada uma prevalência da SAHOS maior em homens (4\%) quando comparado com as mulheres (2\%) (YOUNG et al., 1993).

Os principais fatores de risco para SAHOS são: obesidade (STROBEL; ROSEN, 1996; KYZER; CHARUZI, 1998), gênero masculino (KAPSIMALIS; KRYGER, 2002; SCHWAB, 1999) e envelhecimento (KIM et al., 2004). A obesidade assume papel especial por estar presente em aproximadamente 70\% dos pacientes apnéicos (MALHOTRA; WHITE, 2002) ' e por ser um fator de risco reversível para SAHOS. Em obesos mórbidos, a prevalência pode chegar a $80 \%$ e 50\% nos gênero masculino e feminino, respectivamente (SALVADOR et al., 2004).

O ronco é o sinal mais frequente da doença e definido como um ruído fricativo nos tecidos moles da vias aéreas superiores, pela vibração da úvula e palato, ocasionalmente da parede lateral faríngea, epiglote e base da língua durante o sono, o ronco também é um sintoma constante que pode indicar a presença de SAHOS, com prevalência de $28 \%$ das mulheres e $44 \%$ dos homens, tendendo a aumentar com a idade, elevando-se para $73 \%$ das mulheres e $84 \%$ dos homens na sétima e oitava décadas da vida (ISONO; REMMERS, 1994).

Devido à deficiência que o ar encontra para passar por essa musculatura o organismo vai acumulando o que pode ser chamado de "lixo químico", o qual surge a partir de mudanças no metabolismo que a privação de oxigênio provoca, é formado por uma série de substâncias altamente lesivas às células, principalmente às membranas celulares do organismo, são as espécies reativas de oxigênio ou simplesmente os radicais livres de oxigênio, os portadores de SAHOS são verdadeiras fábricas noturnas desses radicais livres que são moléculas orgânicas e inorgânicas e átomos que contêm um ou mais elétrons não pareados, com existência independente, essa configuração faz dos radicais livres de oxigênio moléculas altamente instáveis, com meia-vida curtíssima e quimicamente muito reativas. A presença dos radicais é crítica para a manutenção de muitas funções fisiológicas normais tais como Hipertensão Arterial Sistêmica (HAS) que está presente em 40\% a 90\% dos pacientes com SAHOS, ritmo cardíaco e patologias como Infarto Agudo do Miocárdio, Insuficiência Cardíaca Congestiva, Acidente Vascular Encefálico e até a morte quando associada a obesidade e Hipertensão Arterial Sistêmica (ALOÉ, 1997; CINTRA et al., 2006; REIMÃO; JOO, 2000).

O exame considerado padrão ouro para diagnóstico da SAHOS é a polissonografia, que é um termo genérico que se refere ao registro simultâneo de algumas variáveis fisiológicas durante o sono, reunindo vários exames em um só, tais como eletrocardiograma (EGG), eletrooculograma (EOG), eletromiografia (EMG), movimento do tórax e abdome e temperatura corporal, dentre outros (DRAGER et al., 2005).

A gravidade da doença, de acordo com o consenso da American Academy of Sleep Medicine Task Force foi baseada nos critérios de sonolência excessiva diurna, o principal parâmetro na SAHOS é o número de eventos de apneia e hipopneia por hora de sono, sendo o este considerado o principal índice. Define-se como normal quando o número de eventos de apneia ou hipopeneia é inferior a cinco, como leve entre cinco e quinze, como moderado quinze a trinta e como grave quando maior que trinta eventos por hora de sono (AMERICAN ACADEMY OF SLEEP MEDICINE, 1999).

A QV dos portadores de SAHOS tem um declínio significativo, diminuição da libido e impotência sexual ocorre em cada três homens que roncam, alterações de personalidades como irritabilidade, agressividade e ansiedade podem estar presente causando sofrimento individual, até mesmo a perda do parceiro que devido o barulho estridente dos roncos preferem dormir em quartos separados causando assim posteriormente separação (ALOÉ, 1997). Pensando em todos esses transtornos foi desenvolvida e aplicada uma intervenção sobre a higiene do sono para avaliar se a QV dos portadores de SAHOS melhora após a mesma.

Considerando o contexto apresentado, esta pesquisa teve como objetivo avaliar a QV dos pacientes com SAHOS após um programa de intervenção para a melhora do sono. 


\section{Método}

O projeto de pesquisa foi aprovado pelo Comitê de Ética em Pesquisa do Hospital Geral do Grajaú sob o protocolo $n^{\circ}$ 041/2009. A pesquisa teve vinculação à Disciplina de Trabalho de Conclusão do Curso de Graduação em Enfermagem da Universidade de Santo Amaro, São Paulo, SP.

Tratou-se de uma pesquisa quase-experimental (LoBIONDO-WOOD; HABER, 2001) do tipo antes-depois, realizada com pacientes com diagnóstico médico de SAHOS cadastrados no Ambulatório Médico de Especialidades Interlagos, em São Paulo, SP. Devido às limitações do serviço, somente ingressam nas atividades terapêuticas dez pacientes a cada vez. Dois sujeitos, um de cada grupo foram excluídos por falta de adesão ao protocolo. $\mathrm{O}$ estudo ocorreu de setembro a dezembro de 2009.

Os nove primeiros pacientes da lista foram convidados a fazer parte do estudo sobre a higiene do sono. Sem randomização, este grupo constitui-se no Grupo Experimental (GE). Outro grupo dos próximos nove pacientes foi abordado para participar do estudo, constituindo-se na formação do Grupo Controle (GC). Para ambos os grupos foram considerados os seguintes critérios: ter realizado a polissonografia, não ter sequelas neurológicas com comprometimento de marcha, não ter alterações cardiovasculares e não estar realizando qualquer intervenção para melhora do sono.

Para ambos os grupos, a abordagem constitui-se da apresentação oral da pesquisa em uma reunião e apresentação da carta de informação, seguida da assinatura do Termo de Consentimento Livre e Esclarecido. Esta composição usou a técnica não-probabilística de conveniência.

Previamente às intervenções, ambos os grupos foram submetidos ao Questionário multidimensional da avaliação da QV SF-36 (WARE; SHERBONE, 1992), originalmente na língua inglesa e que no Brasil teve sua tradução e validação cultural realizada por Ciconelli (1997). O instrumento é constituído de 36 itens, fornecendo pontuação em oito dimensões da QV: capacidade funcional (CF), Limitação por aspectos físicos (AF), dor (DF), estado geral de saúde (SG), vitalidade (VIT), aspectos sociais (AS), aspectos emocionais (AE) e saúde mental (SM). A pontuação varia de 0 (pior resultado) a 100 (melhor resultado), antes e depois da intervenção.

O GC teve uma reunião/palestra de apresentação do programa com informações sobre a fisiopatologia da SAHOS e como eles seriam tratados pelos demais profissionais, sem receber a intervenção sobre a higiene do sono.

O início da intervenção sobre a higiene do sono para o GE foi por meio de uma palestra de 50 minuotos com informações sobre a fisiologia do sono, da musculatura oro-facial, as causas da apnéia e suas consequências. Na sequencia, outros 11 encontros de 30 minutos foram realizados nas semanas seguintes tendo as seguintes temáticas:

- Procure manter horários regulares para se deitar e acorda (não deixar que hábitos irregulares virem uma rotina);

- Faça exercícios regularmente (os exercícios favorecem o chegar a cama relaxado, tanto física com mentalmente quanto fisicamente);

- Transforme em ocupação suas preocupações (um grande erro é deitar e ficar pensando compulsivamente em tudo o que aconteceu durante o dia);

- Cuide de sua alimentação ( as comidas pesadas podem causar grandes desconfortos);

- Evite consumo de bebidas alcoólicas durante à noite (o álcool relaxa a musculatura e facilita o surgimento dos roncos);

- Evite fumar à noite (a nicotina é um estimulante ainda mais poderoso do que a cafeína), Evite "brigar" com a cama (o sono sempre quer chegar, basta nos preparar para ele);

- Cuide de seu nariz (aproveite o momento do banho para fazer uma boa higiene das narinas);

- Se necessário, desenvolva um ritual de sono (escutar música, tomar um banho, ler um bom livro); 
- Não usar remédios para dormir por conta própria( usar medicações somente com orientação médica);

- Durma o tempo suficiente para se sentir bem (procure uma boa qualidade de sono e não quantidade);

- Mantenha a tranquilidade do local onde você dorme (evite assistir TV, estudar ou jogar videogame);

- Mantenha um ambiente adequado ao sono (sons, luminosidade, temperatura, podem interferir na qualidade do sono).

Para a análise dos resultados foram aplicados os seguintes testes: análise de variância de Friedman (SIEGEL; CASTELLAN JÚNIOR, 2006) com o objetivo de comparar os oito domínios da QV SF-36 em relação às diferenças percentuais $(\Delta \%)$ calculados a partir das diferenças entre os valores observados no pré e pós-intervenção. Para o cálculo do $\Delta \%$ aplicou-se a fórmula: $\Delta \%=$ ((valor pós - valor pré)/ valor pré)/100. Esta análise foi aplicada em separado para ambos os grupos. Outra análise incluiu o teste de Mann-Whitney (SIEGEL; CASTELLAN JÚNIOR, 2006) para comparar os GC e GE em relação às diferenças anteriormente assinaladas. Este teste foi aplicado em separado para cada um dos domínios.

\section{Resultados}

\subsection{Perfil da Amostra}

Participaram da pesquisa dois grupos de pacientes portadores de SAHOS, sendo ambos constituídos por nove pacientes. Quanto à idade, a faixa etária de maior frequência foi a de 40 a 50 anos, 55,6\% no GC e 44,5\% no GE. Todas as demais faixas tiveram menor frequência, tendo um ou dois sujeitos.

Quanto ao sexo, em ambos os grupos houve predominância de sujeitos do sexo masculino, no GC 66,7\% (6) e no GE 55,6\% (5). Outra variável estudada foi o IMC (Índice de Massa Corpórea), categorizado em menor do que 25 e maior do que 25 . No GC 55,6\% encontravam-se na faixa de sobrepeso e no GE 66,7\%. Maiores detalhes estão apresentados na Tabela 1:

Tabela 1 - Variáveis demográficas dos grupos estudados (GC=9 e GE=9). São Paulo, 2010.

\begin{tabular}{|c|c|c|c|c|}
\hline \multirow{2}{*}{ Variáveis } & \multicolumn{2}{|c|}{ Grupo Controle } & \multicolumn{2}{|c|}{ Grupo Experimental } \\
\hline & $\mathbf{n}$ & $\%$ & $\mathbf{n}$ & $\%$ \\
\hline \multicolumn{5}{|l|}{ Faixas etárias } \\
\hline De $40-50$ & 5 & 55,6 & 4 & 44,5 \\
\hline De $51-60$ & 2 & 22,2 & 1 & 11,1 \\
\hline De $61-70$ & 2 & 22,2 & 1 & 11,1 \\
\hline De $71-80$ & - & 0,0 & 2 & 22,2 \\
\hline Mais de 80 & - & 0,0 & 1 & 11,1 \\
\hline \multicolumn{5}{|l|}{ Sexo } \\
\hline Feminino & 3 & 33,3 & 4 & 44,4 \\
\hline Masculino & 6 & 66,7 & 5 & 55,6 \\
\hline \multicolumn{5}{|l|}{$I M C$} \\
\hline$<25$ & 4 & 44,4 & 3 & 33,3 \\
\hline$>25$ & 5 & 55,6 & 6 & 66,7 \\
\hline
\end{tabular}

Fonte: Pesquisa de campo (2009)

\subsection{Teste da QV relacionada ao sono}

O instrumento SF-36 foi aplicado igualmente para ambos os grupos antes das intervenções. O GC recebeu as orientações habituais e o GE a intervenção sobre a higiene do sono. Depois das intervenções, o SF-36 foi novamente aplicado a ambos os grupos. Os resultados demonstraram que o GE obteve valores superiores ao GC (Tabela 2): 
Tabela 2 - QV relacionada ao sono por domínios antes e depois da intervenção

\begin{tabular}{|c|c|c|c|c|c|}
\hline \multirow{3}{*}{ Domínios } & \multicolumn{4}{|c|}{ Escores } & \multirow{3}{*}{$p$ valor* } \\
\hline & \multicolumn{2}{|c|}{ GC } & \multicolumn{2}{|c|}{ GE } & \\
\hline & Antes & Depois & Antes & Depois & \\
\hline Capacidade funcional & 73,8 & 75,0 & 67,7 & 84,4 & \multirow{8}{*}{$>0,05$} \\
\hline Limitação por aspectos físicos & 77,2 & 74,4 & 63,9 & 80,5 & \\
\hline Dor & 53,8 & 55,05 & 58,7 & 86,4 & \\
\hline Estado geral de saúde & 52,3 & 52,72 & 50,7 & 59,2 & \\
\hline Vitalidade & 50,0 & 53,33 & 41,6 & 46,6 & \\
\hline Aspectos sociais & 66,6 & 65,27 & 66,6 & 79,0 & \\
\hline Limitações por aspectos emocionais & 88,8 & 85,13 & 59,1 & 85,1 & \\
\hline Saúde mental & 51,5 & 52,4 & 46,2 & 56,8 & \\
\hline
\end{tabular}

*Teste de Mann-Whitney

Fonte: Pesquisa de Campo (2009)

As médias das pontuações referentes à QV nas dimensões/domínios englobados pelo instrumento SF-36 foram: Capacidade funcional=U $16(\mathrm{p}>0,05)$; Limitação por aspectos físicos $=\mathrm{U}$ $16(\mathrm{p}>0,05) ;$ Dor = U $19(\mathrm{p}=>0,05)$; Estado geral de saúde= U $27(\mathrm{p}>0,05) ;$ Vitalidade= U $33(\mathrm{p}$ $>0,05)$; Aspectos sociais $=\mathrm{U} 25(\mathrm{p}>0,05)$; Limitação por aspectos emocionais $=\mathrm{U} 17(\mathrm{p}>0,05)$; Saúde mental $=$ U 22,5 ( $\mathrm{p}>0,05)$.

No teste de Wilcoxon as variâncias foram: Capacidade funcional no $\mathrm{GC}=16(\mathrm{p}>0,05)$, $\mathrm{GE}=1,50(\mathrm{p}<0,01)$; Limitação por atividade física no $\mathrm{GC}=0,5(\mathrm{p}<0,01), \mathrm{GE}=0,3(\mathrm{p}=<0,01)$; Dor no $\mathrm{GC}=1,5$ ( $\mathrm{p}<0,01), \mathrm{GE}=1(\mathrm{p}<0,01)$; Estado geral de saúde no $\mathrm{GC}=10,5(\mathrm{p}>0,05), \mathrm{GE}=3$ ( $\mathrm{p}$ $>0,05)$; Vitalidade no $\mathrm{GC}=2,5(\mathrm{p}>0,05), \mathrm{GE}=9(\mathrm{p}>0,05)$; Limitação por aspectos sociais no $\mathrm{GC}=2$ $(p=0,01), G E=2(p=0,01)$; Aspectos emocionais no $G C=2,5(p>0,05), G E=3,5(p>0,05)$ e Saúde mental no $\mathrm{GC}=9,5(\mathrm{p}>0,05), \mathrm{GE}=4,5(\mathrm{p}>0,05)$.

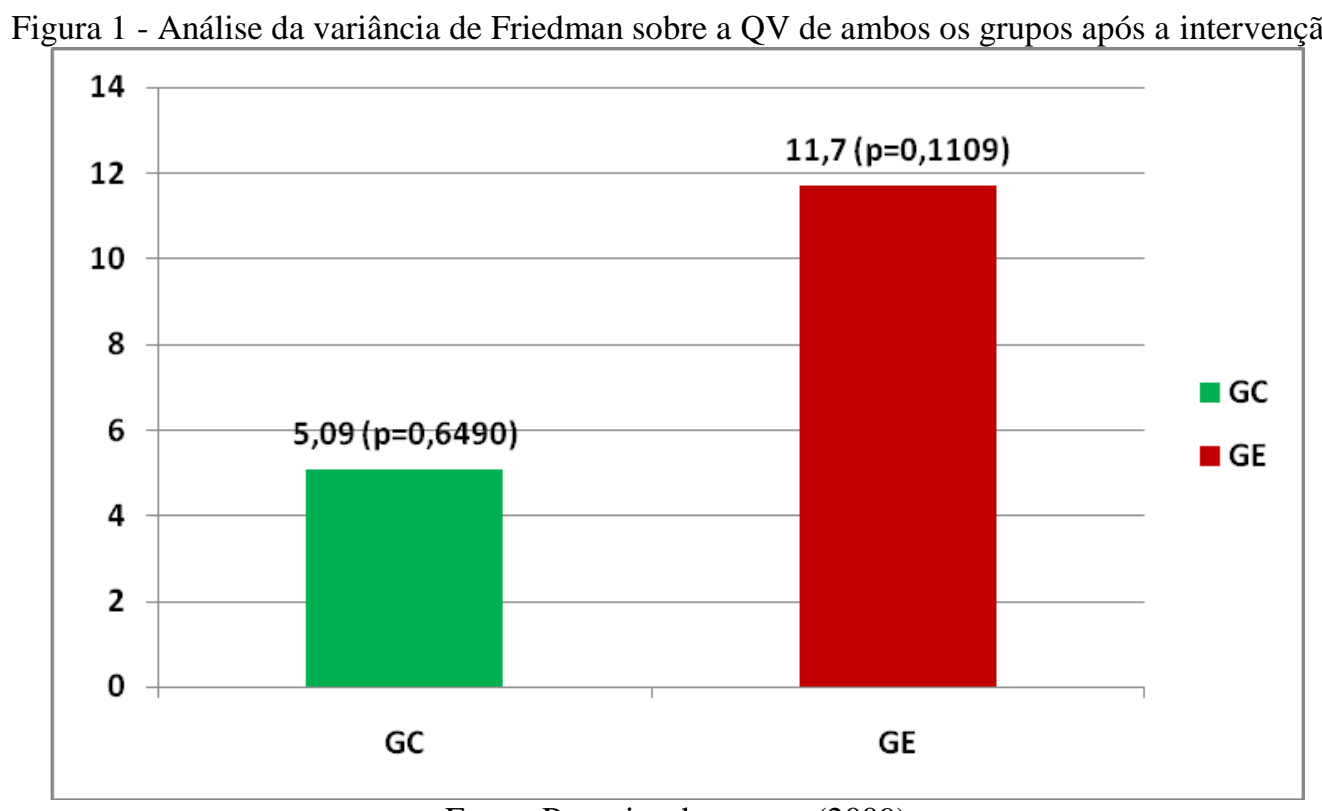

Fonte: Pesquisa de campo (2009)

\section{Discussão}

Os dados apontados na pesquisa demonstraram abrangência e podem ser direcionados a várias perspectivas. $\mathrm{O}$ teste sobre a $\mathrm{QV}$ relacionada ao sono ao qual foram submetidos os 18 sujeitos constatou que os resultados apresentaram significância. Em todos os domínios houve resultados positivos para o GE. A maioria dos sujeitos encaminhados para terapia de intervenção pertenceu ao gênero masculino, como era esperado, já que esse gênero é conhecido como fator de risco para SAHOS (MOHSENIN, 2003; JORDAN; McEVOY, 2003). A maior parte desses pacientes apresentou-se com sobrepeso $(61,1 \%)$ e obesidade $(36,3 \%)$ e ainda pode-se notar o 
aumento da frequência e gravidade da SAHOS à medida que aumentava o IMC. A relação da SAHOS com a obesidade já está bem estabelecida (ISONO; REMMERS, 1994), e esse estudo vem corroborar com a literatura nesse aspecto, ao demonstrar que a variável obesidade foi a maior preditora para SAHOS. Muito embora essa associação esteja clara, os mecanismos por meio dos quais isso acontece, ainda não estão bem estabelecidos (KOENIG, 2001). A literatura sugere que o excesso de peso levaria ao estreitamento da faringe, ou por deposição de gordura nas paredes da faringe, como língua, palato mole e úvula (KOENIG, 2001; SHELTON et al., 1993). Além do mais, vários estudos têm sugerido que a presença de gordura ao redor da faringe aumenta a complacência da mesma, favorecendo o colapso durante o sono (KOENIG, 2001; STROHL; STROBEL; PARISI, 1998).

A dimensão de QV com pontuações mais baixas (piores resultados) foram Dor, Saúde geral e Vitalidade. Esse resultado é concordante com a literatura nacional, que demonstra predomínio de pontuações mais baixas obtidas pelo SF-36 referentes à dimensão limitação por aspectos físicos entre pacientes com SAHOS (STROHL; STROBEL; PARISI, 1998). A média de idade dos sujeitos participantes do presente estudo mostra que os mesmos estão distribuídos na faixa potencialmente produtiva; ou seja, de 40 a 50 anos no GC (55,6\%) e no GE (44,5\%); entre 51 a 60 anos no GC $(22,2 \%)$ e no GE $(1,1 \%)$. Este resultado pode explicar a baixa ocupação profissional e por tornar-se alvo de máxima atenção por parte dos profissionais de saúde envolvidos no cuidado desses pacientes.

Cerca de $66,3 \%$ dos sujeitos da amostra relataram melhora na dor no corpo e principalmente diminuição da cefaleia, este último pode estar associada com a hipertensão, já que $86,2 \%$ dos sujeitos deste estudo disseram ser hipertensos, isto vai de encontro com estudos que verificaram que a prevalência de HAS em pacientes com SAHOS é de $40 \%$ a $90 \%$, e o inverso, a prevalência da SAHOS entre portadores de HAS, é de 22\% a 62\% (CINTRA et al., 2000). O GE mostrou melhora em realizar simples tarefas, entre elas agachar, vestir-se e caminhar sem dificuldade. Segundo Reimão e Joo (2000), os distúrbios do podem causar fadiga, falta de energia e dores de cabeça quando comparados a pessoas com sono normal. A Limitação por aspectos físicos abrange dedicação a tarefas, prazer em realizar atividades e dificuldade no trabalho. O GC mostrou variação mínima enquanto o GE teve significativa melhora. Os pacientes voltaram a realizar atividades que antes não realizavam.

\section{Conclusões e recomendações}

Após o oferecimento da intervenção sobre a higiene do sono, pode-se constatar estaticamente que o GE teve melhora na QV em todos os domínios abordados no instrumento SF36, com apresentação de significância estatística.

Os domínios com maior diferença estatística no GE foram Capacidade Funcional com médias do Questionário QV SF-36 de 67,7 no teste pré e 84,4 no teste pós-intervenção ( $\mathrm{p}=>0,05)$, Limitação por aspectos físicos 63,9 no teste pré e 80,5 no teste pós-intervenção $(\mathrm{p}=>0,05)$ e Dor 58,7 no teste pré e 86,4 no teste pós-intervenção $(\mathrm{p}=>0,05)$ e Limitação por aspectos emocionais 59,1 no teste pré e 85,1 no teste pós-intervenção $(\mathrm{p}=>0,05)$. Este último com destaque maior, já que a intervenção sobre higiene do sono atuou diretamente nesta limitação, pois nas reuniões semanais eram discutidos vários aspectos emocionais que impediam os pacientes de terem uma boa noite de sono. Isto colaborou para confirmar o grau de importância das informações oferecidas na intervenção sobre a higiene do sono, ressaltando que em grupos anteriores de pacientes com SAHOS a não foi oferecida a intervenção sobre a higiene do sono.

A recomendação produzida por este estudo é que haverá benefício aos pacientes portadores de SAHOS com a adição das informações disponibilizadas pela intervenção sobre a higiene do sono a todos os pacientes que ingressarem no tratamento. 


\section{Referências}

ALOÉ, F. Ronco e apnéia obstrutiva do sono. Revista Fono Atual, São Paulo, v. 1, n. 1, p. 34-39, 1997.

AMERICAN ACADEMY OF SLEEP MEDICINE. Sleep-related breathing disorders in adults: Recommendations for syndrome definition and measurement techniques in clinical research. Sleep, Darien, v. 22, n. 5, p. 667-689, sep. 1999.

DRAGER, L. F. Early signs of atherosclerosis in Obstructive Sleep Apnea. American Journal of Respiratory and Critical Care Medicine, New York, v. 172, n. 5, p. 613-618, sep. 2005.

CICONELLI, R. M. Traducao para o portugues e validacao do questionário genérico de avaliação de qualidade de vida "medical outcomes study 36-item short-form health survey (SF-36). 1997. 145 f. Tese (Doutorado em Medicina) - Escola Paulista de Medicina, Universidade Federal de São Paulo, São Paulo.

CINTRA, F. D. et al. Alterações cardiovasculares na síndrome da apnéia obstrutiva do sono. Arquivos Brasileiros de Cardiologia, São Paulo, v. 86, n. 6, p. 191-197, jun. 2006.

ISONO, S.; REMMERS, J. E. Anatomy and physiology of upper airway obstruction. In: KRIEGER, M.H.; ROTH, D.W.C. (Org.). Principles and practices of sleep medicine. 3. ed. Philadelphia: WB Saunders, 1994. p. 642-656.

JORDAN, A. S.; McEVOY, R. D. Gender differences in sleep apnea: Epidemiology, clinical presentation and pathogenic mechanisms. Sleep Medicine Reviews, New York, v. 7, n. 3, p. 373376, oct. 2003.

KAPSIMALIS F.; KRYGER, M. H. Gender and obstructive sleep apnea syndrome, part 1: Clinical features. Sleep, Darien, v. 25, n. 4, p. 412-419, jun. 2002.

KIM, J. et al. Prevalence of sleep-disordered breathing in middle-aged Korean men and women. American Journal of Respiratory and Critical Care Medicine, New York, v. 170, n. 10, p. 1108-1013, nov. 2004.

KOENIG, S. M. Pulmonary complications of obesity. American Journal of Medical Sciences, Philadelphia, v. 321, n. 4, p. 249-279, apr. 2001.

KYZER, S.; CHARUZI, I. Obstructive sleep apnea in the obese. World Journal of Surgery, Philadelphia, v. 22, n. 9, p. 998-1001, sep. 1998.

LoBIONDO-WOOD, G.; HABER, J. Pesquisa em enfermagem: métodos, aplicação e avaliação crítica. 3. ed. Rio de Janeiro: Guanabara-Koogan, 2001.

MALHOTRA, A.; WHITE, D. P. Obstructive sleep apnea. Lancet, New York, v. 360, n. 9328, p. 237-245, jul. 2002.

MOHSENIN, V. Effects of gender on upper airway collapsibility and severity of obstructive sleep apnea. Sleep Medicine, New York, v. 4, n. 6, p. 523-529, nov. 2003.

PAKOLA, S. J. Driving and sleepiness review of regulation and guidelines for commercial and noncommercial drivers with sleep apnea and narcolepsy. Sleep, Darien, v. 18, n. 5, p. 787-796, nov. 
1995.

REIMÃO, R.; JOO, S. H. Mortalidade da apnéia obstrutiva do sono. Revista da Associação Médica Brasileira, São Paulo, v. 46, n. 1, p. 52-56, jan./mar. 2000.

SALVADOR, J. et al. The obstructive sleep apnea syndrome in obesity: A conspirator in the shadow. Revista Medica de la Universidad de Navarra, Navarra, v. 48, n. 1, p. 48-62, jan./mar. 2004.

SCHWAB, R. J. Sex differences and sleep apnea. Thorax, London, v. 54, n. 2, p. 284-285, mar./apr. 1999.

SHELTON, K. E. et al. Pharyngeal fat in obstructive sleep apnea. American Reviews in Respiratory Diseases, New York, v. 143, n. 2, p. 462-466, aug. 1993.

SIEGEL, S. E.; CASTELLAN JÚNIOR, N. J. Estatística não-paramétrica para ciências do comportamento. Porto Alegre: Artmed, 2006.

STROBEL, R. J; ROSEN, R. C. Obesity and weight loss in obstructive sleep apnea: a critical review. Sleep, Darien, v. 19, n. 2, p. 104-115, feb. 1996.

STROHL, K. P.; STROBEL, R. J.; PARISI, R. A. Obesity and pulmonary function. In: BRAY, G. A.; BOUCHARD, C.; JAMES, W. P. T. (Org.). Handbook of obesity. New York: Marcel Dekker, 1998. p. 725-739.

WARE, J. E.; SHERBONE, C. D. The MOS 36-Item short- Form Health Survey (SF-36). Conceptual framework and item selection. Medical Care, New York, v. 30, n. 6, p. 473-483, jun. 1992.

YOUNG, T. et al. The occurrence of sleep-disordered breathing middle-aged adults. New England Journal of Medicine, New York, v. 328, n. 17, p. 1230-1235, apr. 1993. 